\title{
« et après quand il a réussi à rentrer à la maison/ sa maman elle/ elle lui a fait une tisane de camomille »
}

Sur les procédures d'apprentissage du lexique : reprise et reformulation en langage oral à l'école maternelle

"And after when he managed to return home/his mom she/she made him a herb tea of camomile". On the procedures of learning of the lexicon: resumption and reformulation in oral language to the nursery school

\section{Pierre Péroz}

\section{(2) OpenEdition}

Édition électronique

URL : http://journals.openedition.org/pratiques/4427

DOI : $10.4000 /$ pratiques. 4427

ISSN : 2425-2042

Éditeur

Centre de recherche sur les médiations (CREM)

Référence électronique

Pierre Péroz, « « et après quand il a réussi à rentrer à la maison/ sa maman elle/ elle lui a fait une tisane de camomille » », Pratiques [En ligne], 177-178 | 2018, mis en ligne le 20 juillet 2018, consulté le 20 avril 2019. URL : http://journals.openedition.org/pratiques/4427 ; DOI : 10.4000/pratiques.4427

Ce document a été généré automatiquement le 20 avril 2019.

(c) Tous droits réservés 


\section{" et après quand il a réussi à rentrer à la maison/ sa maman elle/ elle lui a fait une tisane de camomille »}

Sur les procédures d'apprentissage du lexique : reprise et reformulation en langage oral à l'école maternelle

"And after when he managed to return home/his mom she/she made him a herb tea of camomile". On the procedures of learning of the lexicon: resumption and reformulation in oral language to the nursery school

Pierre Péroz

\section{Introduction}

1 «Comment les élèves apprennent à parler à l'école » était le thème proposé à notre réflexion. Les Programmes $2015^{1}$ comme le faisaient déjà ceux de $2008^{2}$ font de la parole magistrale l'origine essentielle des apprentissages langagiers et linguistiques des élèves. Rappelons le premier paragraphe consacré au domaine du langage oral dans les Programmes 2015 :

«L'enfant, quelle que soit sa langue maternelle, dès sa toute petite enfance et au cours d'un long processus, acquiert spontanément le langage grâce à ses interactions avec les adultes de son entourage. L'enseignant, attentif, accompagne chaque enfant dans ses premiers essais, reprenant ses productions orales pour lui apporter des mots ou des structures de phrase plus adaptés qui l'aident à progresser. L'enseignant s'adresse aux enfants les plus jeunes avec un débit ralenti de parole ; il produit des énoncés brefs, syntaxiquement corrects et soigneusement articulés. »

2 Le modèle sous-jacent est explicitement celui de l'apprentissage du langage dans «la toute petite enfance " où les interactions individualisées sont naturellement la norme. Nous nous sommes déjà interrogé sur la pertinence de ce modèle "dialogal adultocentré » en contexte scolaire (Péroz, 2015). Il y a là une conception modélisante de 
l'enseignement du langage oral à l'école qui nous parait discutable par rapport à ce que nous savons des procédures d'apprentissage dont témoigne le corpus CLEA sur lesquelles nous allons revenir au cours de cette étude.

En effet, non seulement l'essai, la reprise ou la reformulation par les élèves eux-mêmes nous paraissent constitutifs de l'apprentissage mais ils nous paraissent aussi appartenir à la nature même du langage et du langage oral en particulier. Aussi faisons-nous l'hypothèse, après F. François (1993), que la réussite de son apprentissage tient justement aux possibilités de "reprise-modification », et plus largement d'enchainements dans la « continuité et/ou le mouvement » que la séance offre à l'élève. Pour le montrer, et parce qu'il fallait ici délimiter notre propos, nous nous intéresserons plus particulièrement aux apprentissages lexicaux.

4 Un survol de la littérature existante sur le sujet montre qu'il vaudrait mieux nous intéresser à l'apprentissage du lexique dans la langue écrite. Les grandes synthèses de ces dernières années (Nonnon, 2012 ou Grossmann \& Plane, 2008 et Masseron, 2005) témoignent de ce tropisme scriptural dont les raisons sont assez évidentes. D'un autre côté, la plupart de ces travaux montrent que les processus d'engendrement des énoncés, bien plus que leur aboutissement, semblent la piste la plus féconde pour la recherche sur l'acquisition du lexique. C'est ainsi qu'en prenant résolument le point de vue de l'apprentissage plutôt que celui de l'enseignement à l'instar d'auteurs comme E. Calaque et F. Grossmann (2000) ou K. Duvignau et Cl. Garcia-Debanc (2008), nous retrouverons certaines des observations faites sur la langue écrite. Ce qui est plutôt rassurant.

Quelles procédures les élèves mobilisent-ils pour réemployer le vocabulaire présent dans le texte?

6 Pour répondre à cette question, nous avons étudié comment les élèves reprenaient ou reformulaient une cinquantaine de termes ou d'expressions, soit à peu près $10 \% \mathrm{du}$ vocabulaire présent dans le texte initial ${ }^{3}$. Chaque séance ne développant pas toujours les mêmes éléments de l'histoire, nous avons retenu des termes suffisamment variés pour exploiter l'ensemble du corpus. C'est ce que nous verrons dans un premier temps. Dans un deuxième temps, nous verrons quelles sont les procédures de réemploi ${ }^{4}$ mobilisées par les élèves en fonction de leur niveau langagier. Dans un troisième temps, en prenant l'exemple des élèves les moins diserts nous montrerons quelles compétences discursives ils mettent au service de ces apprentissages lexicaux. Pour conclure, nous verrons quelles conséquences didactiques on peut tirer de ces observations.

\section{Le corpus d'étude et les élèves}

\section{Le choix des termes étudiés}

7 Le choix des termes a répondu à des critères croisés qui ont d'abord été leur présence dans les principales péripéties de l'histoire sauf celui de la rencontre avec la chatte observant un poisson qui n'a guère été repris par les élèves. Le tableau suivant montre simplement leur répartition dans le texte. 
L'histoire est celle de quatre adorables petits lapins qui s'appellent Romarin, Capucine, Neige et Pierre. Ils vivent seuls avec leur maman, dans Situation initiale un beau terrier, sous les racines d'un vieux pin centenaire.

Un matin, Maman Lapin dit à ses lapereaux: «Mes chéris, je dois m'absenter pour la journée. Vous pouvez jouer dans le pré mais, surtout, surtout n'allez pas dans le jardin de monsieur Grégory! Votre pauvre père n'en est jamais revenu! Madame Grégory a fait de lui un civet pour son repas. N'approchez pas de ce potager et soyez sages!»

Romarin, Capucine et Neige, qui sont très obéissants, vont dans le pré cueillir des mûres pour le diner. Quant à Pierre, qui est le plus intrépide de la famille, il ne peut retenir son envie d'aller voir le potager interdit de monsieur Grégory. Arrivé devant, il se faufile sous la barrière, sans la moindre inquiétude. Il n'en croit pas ses yeux! Un potager de rêve! Quel festin! Tout d'abord il goûte quelques belles laitues bien croquantes, puis mange deux ou trois gros haricots verts et d'énormes radis. Il dévore tout ce qu'il veut et trouve cela bien amusant et, ma foi, pas aussi dangereux qu'a prétendu sa maman! Comme il a trop mangé, il déguste dans un coin un bouquet de persil pour digérer calmement.

Alors qu'il termine tranquillement un concombre, un bruit l'effraie. Un bruit étrange et inquiétant. Que se passe-t-il tout à coup dans ce potager si tranquille? C'est monsieur Grégory qui vient de l'apercevoir alors qu'il est en train de planter des jeunes choux. Il se lève d'un bond et court vers Pierre en hurlant : «Par mes moustaches, arrête! Petit voleur de lapin! Je vais t'attraper et te transformer en pâté ! » Pierre est terrifié, il se met à courir, dans tous les sens, sans pouvoir retrouver l'endroit par lequel il est entré ! Il perd une chaussure dans les choux et l'autre dans les pommes de terre.

Il court ventre à terre aussi vite qu'il le peut. Il est essoufflé, épuisé. Mais c'est alors que le bouton de son gilet se prend dans le filet qui recouvre le plant de groseilles qu'il est en train de traverser. Catastrophe! Il regarde autour de lui, les oiseaux qui l'observent lui crient de se sauver! Monsieur Grégory arrive, un panier à la main, prêt à l'attraper. Vite, vite, Pierre ! OUF! Juste à temps! Pierre vient d'enlever son gilet! Il reprend sa course folle, passant juste sous les jambes de monsieur Grégory.

Il arrive au fond du jardin et entre dans la remise. Sans réfléchir, il saute dans l'arrosoir qui se trouve près de lui. Quelle erreur ! Il est à moitié plein d'une eau glacée! Cependant, il ne bouge pas car monsieur Grégory approche. Il le cherche attentivement, partout. Pierre grelotte de peur et de froid et, tout à coup : "Atch... atch... atchoum !" Il éternue si fort que monsieur Grégory se retourne immédiatement vers lui.

Obéissance et transgression. Les plaisirs défendus

Première péripétie. Juron et menace

Deuxième péripétie. Pris au piège! du filet à groseilles Troisième péripétie. L'arrosoir plein d'eau glacée. Quelle erreur. 
Pierre est épuisé, gelé et terrifié. Il s'échappe à nouveau. La course folle reprend de plus belle; il cherche désespérément à sortir du potager. Il arrive près d'une mare, où une chatte blanche observe un poisson rouge dont elle ferait bien son repas. Sa queue frétille d'impatience et Pierre n'ose pas la déranger, ne sachant pas si, comme le lui a dit son cousin, les chats sont vraiment les ennemis des lapins!

Pierre ne s'arrête de courir qu'une fois arrivé dans la douceur de son terrier, sous les racines du vieux pin. Il s'effondre, ferme les yeux et s'endort, épuisé. Sa maman est en train de préparer le repas. Elle se demande pourquoi son petit Pierre a encore perdu ses vêtements et ses chaussures. C'est la deuxième fois, cette semaine! Que fait-il donc?

Le soir même, Pierre ne se sent pas bien. Il est encore perturbé par les émotions de la journée. Sa maman le met au lit et lui donne une tisane de camomille. "Je ne sais pas ce que tu as, mais, avec ça, tu passeras une bonne nuit ! » Pierre ne raconte rien à sa maman mais se jure de ne jamais plus désobéir! Quant à Romarin, Capucine et Neige, ils se partagent les Quatrième péripétie. La rencontre avec la chatte blanche. mûres qu'ils ont ramassées, ainsi que le pain et le lait que leur mère a ramenés pour le diner!

8 Un autre critère a été leur variété syntaxique. Nous avons retenu 47 mots à sens plein ${ }^{5}$, soit $20 \%$ des mots de ce type dans le texte). Certains d'entre eux apparaissent dans le texte dans des expressions ou des constructions verbales qui sont reprises ci-après.

- des adjectifs : épuisé, intrépide, méchant, perturbé, (de plus) belle (soit $11 \%$ des adjectifs du texte : 5 sur 38 dont méchant qui n'est pas dans le texte);

- des verbes : obéir, dévorer, effrayer, (n'en jamais) revenir, (ne pas en) croire (ses yeux), passer (une bonne nuit), s'approcher, se faufiler, se prendre, se retourner, se sauver, s'effondrer, s'endormir (soit $15 \%$ des verbes du texte : 13 sur 84$)$;

- des noms : arrosoir, barrière, bruit, camomille, civet, course, émotions, filet, fruit, gilet, goûter, jardin, lapereaux, légume, moustache, mûre, pâté, peur, pin, potager, pré, racine, remise, rêve, terrier, tisane, une fois que, ventre à terre, vêtement (soit $28 \%$ des noms du texte : 29 noms sur 95 dont fruit et légume qui ne sont pas dans le texte);

- inscription dans des expressions ou des constructions verbales : ventre à terre, ne pas en croire ses yeux, n'en jamais revenir, reprendre sa course folle, (la course folle) reprendre de plus belle, ne s'arrêter qu'une fois arrivé.

\section{Les élèves concernés}

9 Les élèves qui sont à l'origine du corpus n'ont pas tous les mêmes compétences. On peut donc s'interroger sur l'impact de ces différences sur l'apprentissage du lexique. Il n'y a guère de doute en effet que le niveau des compétences lexicales soit dépendant des compétences langagières de l'élève (Nonnon, 2012) 6 qui constituent donc un facteur de variation. On considère aussi généralement que les élèves ont recours aux mêmes procédures d'apprentissage comme la reprise et la reformulation; pour F. François, C. Hudelot et E. Sabeau-Jouannet (1984: 22) «le langage est d'abord répétitionmodification ». Jusqu'à quel point ces procédures sont-elles les mêmes pour tous les élèves ? Pour tenter de répondre à cette question, nous avons réparti les élèves sur trois 
niveaux en fonction de leur participation décomptée en nombre de mots prononcés au cours des séances, c'est-à-dire en fonction de leur occupation de l'espace conversationnel au cours de la séance à laquelle ils participent. Au premier niveau, se trouvent les élèves qui dans chaque groupe ont une participation supérieure d'un tiers à la moyenne du groupe, au troisième niveau se trouvent ceux qui ont une participation d'un tiers inférieure à la moyenne des élèves du groupe, enfin au niveau 2 se trouvent ceux qui sont entre les deux, autrement dit dans la moyenne ${ }^{7}$. Les résultats de cette catégorisation se trouvent sur la première ligne (en grisé) du tableau suivant.

Tableau . Nombre d'élèves et d'interventions dans chaque niveau sur l'ensemble des séances

\begin{tabular}{|l|c|c|c|c|}
\hline & Totaux & Niv. 1 & Niv. 2 & Niv. 3 \\
\hline Nombre d'élèves concernés & 103 & 30 & 28 & 45 \\
\hline Nombre d'interventions élèves & 1409 & 709 & 402 & 298 \\
\hline
\end{tabular}

10 Le nombre d'interventions des élèves par niveau, sur la ligne suivante, donne une première image des proportions relatives de leur présence au cours des échanges. Les élèves de niveau 1 produisent la moitié des interventions, environ 700 interventions, tandis que ceux des niveaux 2 et 3 se répartissent l'autre moitié de manière d'ailleurs inégale, respectivement 400 et 300 interventions.

11 Ces différences ne sont guère imputables aux différences d'âge, si l'on considère simplement les élèves en deux classes d'âges, à partir de l'âge médian qui est de 5 ans et 4 mois pour l'ensemble des élèves ${ }^{8}$, on constate que la longueur moyenne de leurs interventions n'est pas si différente que cela. Les élèves les plus jeunes (en rayé sur le graphique) obtiennent d'ailleurs des résultats équivalents, voire meilleurs que ceux de leurs ainés au niveau 2. 
Figure 1. Longueur moyenne des interventions mesurée en nombre de mots $^{9}$ selon l'âge des élèves

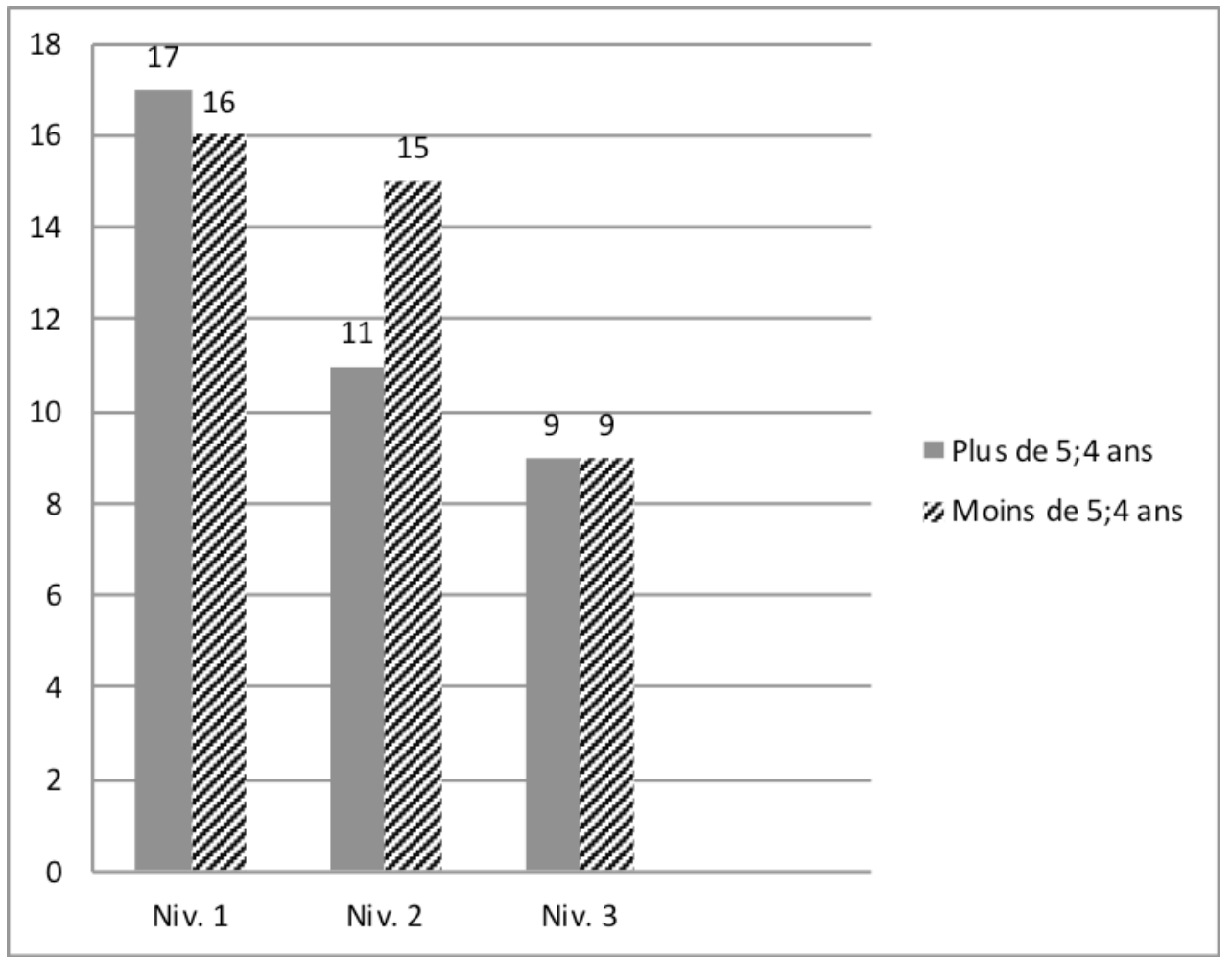

Il nous a donc paru plus pertinent de retenir, pour la suite de nos analyses, ce classement par niveau plutôt qu'un classement par classe d'âge finalement peu représentatif des différences de niveau de compétences entre les élèves.

\section{Analyse du corpus}

Sur les 1409 interventions faites par les élèves ${ }^{10}$, les 47 termes étudiés (désormais TE) ont été repris ou reformulés 1038 fois dans 665 interventions différentes ${ }^{11}$. Comme on pouvait s'y attendre, le taux global de reprise et de reformulation des TE dépend de la participation des élèves aux échanges.

Tableau 3. Pourcentages du nombre des interventions et nombre de termes repris ou reformulés selon le niveau des élèves

\begin{tabular}{|l|c|c|c|}
\hline & Niv. 1 & Niv. 2 & Niv. 3 \\
\hline Pourcentages d'interventions sur l'ensemble du corpus & $50 \%$ & $29 \%$ & $\mathbf{2 1 \%}$ \\
\hline Pourcentages globaux de reprise et de reformulation des TE & $54 \%$ & $28 \%$ & $\mathbf{1 8 \%}$ \\
\hline
\end{tabular}

14 Le nombre d'interventions est important, mais il ne détermine pas exactement le nombre de reprises ou de reformulations des TE. Les élèves de niveau 1, colonne grisée, font $50 \%$ de l'ensemble des interventions et $54 \%$ des réemplois. C'est l'inverse pour les élèves de niveau 3 (en gras), dont le pourcentage de réemplois est inférieur à leur pourcentage d'interventions au cours des séances.

15 Ce tableau appelle donc deux questions. Quelle(s) forme(s) prennent la reprise ou la reformulation des TE et quelles compétences sont-elles mobilisées pour cela? 


\section{Les reprises et les reformulations}

Suivant en cela les travaux de C. Martinot (2010) et de F. Steinbach-Kohler (2008), nous avons considéré aussi bien les «reprises» (le TE est repris tel quel dans l'intervention) que les « reformulations » (le TE est remplacé par un autre mot ou une autre expression) ${ }^{12}$ , même si elles pouvaient paraitre maladroites. Nous faisons ainsi l'hypothèse, conforme au modèle socioconstructiviste de l'apprentissage qui est défendu ici, que ces reformulations ne sont pas différentes sur le plan acquisitionnel des reprises lexicales, voire qu'elles les préparent. Nous avons donc étudié comment les élèves formulaient leurs réemplois des TE en distinguant les «reprises" (au sens strict) des «reformulations». Dans chaque catégorie, nous avons fait la différence entre les formulations qui constituaient des séquences bien formées sémantiquement et syntaxiquement : les reprises ou les reformulations «adaptées » et celles qui semblaient moins bien formées sur un plan ou sur autre: les reprises ou les reformulations « essayées ».

\section{Les reprises}

Dans les reprises adaptées (désormais « Rep-adapt »), les termes sont repris, avec ou non, des modifications qui permettent une intégration correcte dans le contexte de l'intervention. Les élèves étaient amenés, par exemple, à reprendre les mots terrier et pin présents dans le texte original dans la phrase : « Ils vivent seuls avec leur maman, dans un beau terrier, sous les racines d'un vieux pin centenaire ». Dans le passage suivant tandis que Gaël (264) fait une " reprise essayée ", Sandra (268) montre qu'elle a bien compris la place respective du pin mais qu'elle elle ne sait pas vraiment ce qu'est un pin et demande « en dessous du grand pin, c'est quoi un grand pin?»; elle fait ainsi deux «reprises adaptées ».

Tableau 4. Exemple de reprises essayées et adaptées du nom « pin »*

\begin{tabular}{|l|l|l|l|}
\hline Séance & $\mathbf{N}^{\mathbf{0}}$ & Loc & Interventions \\
\hline 2 & 262 & M & ils vivent où/ les lapins \\
\hline 2 & 263 & Sandra & dans leur terrier \\
\hline 2 & 264 & Gaël & dans un grand pin \\
\hline 2 & 265 & M & dans un terrier ou dans un pin \\
\hline 2 & 266 & Soliman & un terrier! \\
\hline 2 & 267 & M & et le pin alors \\
\hline 2 & 268 & Sandra & en dessous du grand pin/ c'est quoi un grand pin \\
\hline 2 & 269 & M & tu poses toujours les mêmes questions Sandra/ \\
\hline 2 & 270 & El(s) & un arbre comme un sapin un peu// \\
\hline
\end{tabular}


* Dans les extraits du corpus comme ci-dessus la première colonne donne le numéro de la séance, la seconde le numéro de l'intervention dans la séance.

Dans les reprises essayées (désormais «Rep-essai »), les termes sont repris mais leur insertion contextuelle est ressentie comme fautive sur le plan sémantique ou syntaxique. Ainsi dans l'exemple suivant, Vicky, après avoir utilisé le mot maison, introduit la séquence (discutable) être sous les racines, sans être tout à faire sûre de sa formulation. On peut penser à une forme involontaire de métonymie, les racines constituant une part du terrier, mais il s'agit quand même d'une reprise essayée.

Tableau 5. Exemple de reprise essayée du nom « racines »

\begin{tabular}{|l|l|l|l|}
\hline Séance & $\mathbf{N}^{\mathbf{0}}$ & Loc & Intervention \\
\hline 9 & 21 & Vicky & $\begin{array}{l}\text { il arrivait à la maison il était il est il est épuisé il est il est euh il était sous } \\
\text { les ranes il s'est endormi et la maman de Pierre elle a donné la maman de } \\
\text { Pierre lui a donné un sirop// un sirop de camomille }\end{array}$ \\
\hline
\end{tabular}

Comme le montrent ces quelques exemples, nous avons pu vérifier ce que plusieurs auteurs (Anctil, 2012 ou Masseron, 2005) qui travaillent sur les processus discursifs soulignaient déjà : lorsqu'un élève reprend un terme, les difficultés ne sont pas vraiment d'ordre sémantique. Par contre, tout ce qui relève de l'ordre syntagmatique peut faire problème : compatibilités lexicales, collocations, expressions idiomatiques, syntaxe et en particulier syntaxe verbale.

21 Ainsi, quand les noms s'inscrivent dans des constructions verbales indirectes, alors leur reprise est difficile, même si ce sont des mots bien connus des élèves. C'est le cas de pâté, inscrit dans la séquence " (te) transformer en pâté », qui donne lieu à plusieurs « reprises essayées». Les erreurs peuvent entre autres porter sur le pronom complément (14), le choix du verbe (42) ou sa construction (26).

Tableau 6. Reprises essayées du nom « pâté »

\begin{tabular}{|l|l|l|l|}
\hline Séances & $\mathbf{N}^{\mathbf{0}}$ & Loc & Interventions \\
\hline 10 & 14 & Ted & $\begin{array}{l}\text { eh ben la maman elle avait dit de pas aller dans le jardin de monsieur } \\
\text { Grégory/ sinon elle va lui en faire un pâté }\end{array}$ \\
\hline 3 & 26 & Melwan & parce que s'il y va/ il va se transformer en pâté \\
\hline 8 & 42 & Sergueï & $\begin{array}{l}\text { Grégory est venu planter les choux et aussi il il a dit/ par mes } \\
\text { moustaches petit lapin voleur je vais te rattraper et te cuisiner en pâté } \\
13 / /\end{array}$ \\
\hline
\end{tabular}

C'est aussi le cas des expressions idiomatiques que les enfants découvrent à l'occasion de ces lectures. Ainsi, l'emploi du verbe reprendre dans les expressions « reprendre sa course folle » et « la course folle reprend de plus belle » pose de sérieux problèmes aux élèves, 
même s'ils comprennent le nom course et sauraient le réemployer dans une construction libre par exemple « faire une course » comme le fait Sonia en $(9,30)$.

Tableau 7. Reprises essayées du nom « course » dans deux expressions idiomatiques

\begin{tabular}{|c|c|c|c|}
\hline Séances & $\mathbf{N}^{\circ}$ & Loc & Interventions \\
\hline 3 & 246 & Yannick & il reprend la course folle \\
\hline 4 & 116 & Thiébaud & $\begin{array}{l}\text { il se rappelle la course folle et il se demande s'il pourrait/ il a failli se } \\
\text { faire attraper }\end{array}$ \\
\hline 5 & 70 & Lili & $\begin{array}{l}\text { les chaussures/ il a perdu une chaussure dans les carottes et une } \\
\text { chaussure dans les choux/// et son gilet/ il a perdu son gilet/ il était } \\
\text { coincé alors il a enlevé son gilet et il a rattrapé sa course// }\end{array}$ \\
\hline 9 & 30 & Sonia & Pierre il a fait une corse(course) une course bien belle \\
\hline 11 & 47 & Akem & $\begin{array}{l}\text { et c'est que que monsieur Grégory il dit arrête/ de par mes } \\
\text { moustaches je vais te transformer en pâté// que la course folle } \\
\text { commence }\end{array}$ \\
\hline
\end{tabular}

\section{Les reformulations}

Nous avons aussi étudié les reformulations des TE par d'autres termes ou expressions que les élèves peuvent trouver pour évoquer la même notion ou le même référent. Là aussi nous avons distingué entre les reformulations pertinentes sur les plans sémantique et syntaxique, les «reformulations adaptées» (désormais «refo-adapt») et les reformulations qui pouvaient poser un problème sur l'un ou l'autre plan, les « reformulations essayées » (désormais « refo-essai »).

Dans la restitution du récit, les élèves étaient amenés à évoquer le retour du héros au domicile familial qui était décrit dans le texte original dans ces termes : «Pierre ne s'arrête de courir qu'une fois arrivé dans la douceur de son terrier...». L'expression verbale restrictive qu'une fois arrivé a donné lieu à des «reformulations adaptées » comme les suivantes.

Tableau 8. Reformulations adaptées du connecteur « ne s'arrête qu'une fois arrivé »

\begin{tabular}{|l|l|l|l|}
\hline Séances & $\mathbf{N}^{\mathbf{o}}$ & Loc & Interventions \\
\hline 10 & 68 & Céline & il a arrêté de courir dès qu'il est: dès qu'il est arrivé dans son terrier \\
\hline 11 & 51 & Théophile & $\begin{array}{l}{[. . .] \text { il s'arrête de courir juste seulement quand il est arrivé dans la }} \\
\text { douceur de son terrier }\end{array}$ \\
\hline
\end{tabular}

Pour les reformulations, l'élève propose un ou d'autres termes à la place du terme étudié, il faut donc que la substitution soit possible en contexte. On trouve alors quelques véritables difficultés lexicales que les élèves contournent souvent dans des 
« reformulations essayées » assez heureuses et inventives, même s'il faut connaitre le texte original pour vraiment apprécier leurs propositions, par exemple pour les noms « civet » et « émotions ».

Tableau . Reformulation essayée de « civet » et de « émotions »

\begin{tabular}{|l|l|l|l|}
\hline Séances & $\mathbf{N}^{\mathbf{0}}$ & Loc & Interventions \\
\hline 3 & 57 & Melwan & ils l'ont chauffé et puis mangé \\
\hline 11 & 55 & Akem & $\begin{array}{l}\text { il il il saute de son fenêtre/ il il rentre dans la maison/ il se met dans son } \\
\text { lit après sa maman lui donne une tisane de camomille comme ça les } \\
\text { trucs qu'il a passés de la journée lui reviennent plus }\end{array}$ \\
\hline
\end{tabular}

\section{Entre essais et adaptation au contexte}

Mais la fréquence et la réussite de ces différentes formes de reprise ou de reformulation ne se bornent pas à la prise en compte des contraintes syntagmatiques. Prenons l'exemple $\mathrm{du}$ nom pré. Le terme n'intervient qu'une fois, au début du texte, dans l'injonction maternelle: "Vous pouvez jouer dans le pré mais, surtout, surtout n'allez pas dans le jardin de monsieur Grégory! ». Pourtant, il donne lieu à 38 occurrences dont 33 reprises alors qu'il ne fait pas partie du vocabulaire des enfants qui habitent des cités pour leur grande majorité, comme le montrent les hésitations des élèves dans les tableaux suivants.

Tableau 10. Hésitations sur l'emploi du nom « pré »

\begin{tabular}{|l|l|l|l|}
\hline Séances & $\mathbf{N}^{\mathbf{0}}$ & Loc & Interventions \\
\hline 2 & 120 & Sandra & $\begin{array}{l}\text { un grand repas où Capucine Neige et Romarin ils mangent les mûres où } \\
\text { ils ont ramassé dans le jardin// dans le pré// c'est un préau? }\end{array}$ \\
\hline 1 & 25 & Oudriss & $\begin{array}{l}\text { eh bien/ après/ après/ après/ Pierre/ Pierre/ il a été dans le champ de } \\
\text { monsieur Grégory }\end{array}$ \\
\hline 4 & 151 & Mélissa & $\begin{array}{l}\text { il a pas écouté sa maman// il a été dans le pré de monsieur Grégory } \\
\text { euh dans le potager de monsieur Grégory }\end{array}$ \\
\hline
\end{tabular}

Tableau 11. Hésitations sur le sens du nom « pré » au cours de la séance 6

\begin{tabular}{|l|l|l|l|}
\hline Séance & $N^{\mathbf{0}}$ & Loc & Interventions \\
\hline 6 & 76 & M & $\begin{array}{l}\text { ça s'appelle comment l'endroit où ils ont été cueillir des mûres près du } \\
\text { sapin? }\end{array}$ \\
\hline 6 & 77 & Céline & la prairie// \\
\hline 6 & 78 & Daly & un pré// \\
\hline
\end{tabular}




\begin{tabular}{|l|l|l|l|}
\hline 6 & 79 & Leslie & $\mathrm{xxx}$ un champ// \\
\hline 6 & 80 & Marco & presque comme un terrier? \\
\hline
\end{tabular}

Mais ces hésitations peuvent être le prélude à son apprentissage. Dans l'histoire, le pré désigne un espace ouvert et permis par opposition à l'espace fermé interdit, celui du potager (ou du jardin). C'est donc en s'appuyant sur cette opposition sémiotiquement pertinente que les élèves reprennent le mot pré, le plus souvent dans la reprise de la recommandation maternelle. Par exemple dans les séances 6, 4 et 3 ci-dessous.

Tableau 12. Reprise de l'opposition « pré »/« jardin » dans la recommandation maternelle

\begin{tabular}{|l|l|l|l|}
\hline Séances & $\mathbf{N}^{\circ}$ & Loc & Interventions \\
\hline 6 & 4 & Marco & $\begin{array}{l}\text { la maman elle dit je dois m'absenter pour la journée/ n'allez pas dans } \\
\text { le purtout surtout n'allez pas dans le jardin de monsieur } \\
\text { Grégory stop }\end{array}$ \\
\hline 4 & 12 & Paulo & $\begin{array}{l}\text { pouvez aller dans le pré/ dans le pré mais surtout surtout n'allez } \\
\text { pas dans le jardin de monsieur Grégory/ votre pauvre père n'en est } \\
\text { jamais revenu// }\end{array}$ \\
\hline 3 & 16 & Marianne & oui/ sa maman il a dit/ vous pouvez \\
\hline 3 & 17 & Malo & d'aller dans le pré/ d'aller dans le pré \\
\hline 3 & 18 & Melwan & et puis son papa il reviendrait jamais parce qu'il s'est fait manger \\
\hline 3 & 19 & Malo & dans le jardin \\
\hline
\end{tabular}

Ainsi, l'analyse des différentes formes de reprise ou de reformulation des TE montre que les paramètres qui interviennent dans la réussite des séquences verbales ainsi constituées touchent à des domaines qui excèdent largement la seule question de leur inscription syntagmatique. S'il y a un point commun à leur trouver, c'est qu'elles se trouvent dans des interventions dont l'enjeu sémantique et interactionnel est clair pour les élèves. C'est la raison pour laquelle, malgré les difficultés que les TE présentent sur le plan syntagmatique les élèves ne les utilisent pas au hasard. Sur les 1038 occurrences répertoriées, en effet, la très grande majorité des reprises ou des reformulations ne pose pas de problèmes particuliers (cases grisées dans le tableau ci-dessous).

Tableau 13. Pourcentages ${ }^{14}$ des différentes formes de reprises ou de reformulations sur l'ensemble des TE

\begin{tabular}{|c|c|c|c|c|}
\hline Rep-adapt. & Rep-essai & Refo-adapt & Refo-essai & Total des Rep-Refo \\
\hline $65 \%$ & $5 \%$ & $20 \%$ & $10 \%$ & $100 \%$ \\
\hline
\end{tabular}




\section{Procédures mobilisées par les élèves selon leur niveau}

Si l'on compare les formes de réemploi par niveau, on constate que les élèves de niveau 1 et 2 ont des résultats assez semblables (voir cellules grisées), avec une légère supériorité des élèves de niveau 1 pour les reprises adaptées.

Tableau 14. Pourcentages des différentes formes de réemploi pour les niveaux 1 et 2

\begin{tabular}{|l|c|c|c|c|l|}
\hline & Rep-adapt & Rep-essai & Refo-adapt & Refo-essai & Total \\
\hline Niv. 1 & $67 \%$ & $5 \%$ & $19 \%$ & $10 \%$ & $100 \%$ \\
\hline Niv. 2 & $66 \%$ & $5 \%$ & $19 \%$ & $10 \%$ & $100 \%$ \\
\hline Niv.3 & $\mathbf{6 0} \%$ & $\mathbf{5 \%}$ & $\mathbf{2 1 \%}$ & $\mathbf{1 4} \%$ & $\mathbf{1 0 0} \%$ \\
\hline
\end{tabular}

30 Les élèves de niveau 3 (en gras) font $6 \%$ ou $7 \%$ de moins de reprises adaptées que leurs camarades de niveau 1 et 2, leur maitrise lexicale est moins assurée. Cela est compensé, par une augmentation à peu près équivalente des reformulations adaptées ou essayées qui leur permettent aussi d'intervenir dans les échanges.

31 Les élèves de niveau 3 par exemple reformulent « dévore tout ce qu'il veut » et autres « radis, haricots verts et laitues » par « manger les / des légumes » dans des contextes qui sont pertinents sur le plan sémantique et interactionnel. Ainsi, à une question de l'enseignant à propos de Pierre qu'on vient de qualifier d'«intrépide », Gaël reprend l'adjectif et le commente en S2-199.

Tableau 15. Reformulations pertinentes sur le plan sémantique et interactionnel

\begin{tabular}{|l|l|l|l|}
\hline Séance & $\mathbf{N}^{\mathbf{0}}$ & Loc & Interventions \\
\hline 2 & 198 & M & $\begin{array}{l}\text { bien bien/ et toi Gaël qu'est-ce que tu penses de Pierre/ il est comment } \\
\text { Pierre }\end{array}$ \\
\hline 2 & 199 & Gaël & intépide// il veut manger tous les légumes de monsieur Grégory// \\
\hline
\end{tabular}

De manière quelque peu elliptique mais pertinente dans ce contexte, Céline justifie l'état de Pierre à son retour à la maison par son passage au jardin de M. Grégory.

Tableau 16. Reformulations pertinentes sur le plan sémantique et interactionnel

\begin{tabular}{|l|l|l|l|}
\hline Séance & $\mathbf{N}^{\mathbf{0}}$ & Loc & Interventions \\
\hline 10 & 26 & Hugues & sa maman elle l'a mis dans dans le lit \\
\hline 10 & 28 & Marjorie & il était énervé \\
\hline 10 & 30 & Céline & $\mathbf{x x x}$ il a mangé tous les légumes \\
\hline
\end{tabular}

33 Comme les élèves des autres niveaux, ils ne reprennent pas la séquence restrictive «Pierre ne s'arrête de courir qu'une fois arrivé dans [...] son terrier». Ils en 
connaissent pourtant le sens et l'importance dans le récit. Voici comment, en poursuivant la narration de Shona (niveau 1), Oudriss (niveau 3) reformule cette séquence en S1-11 et justifie sa proposition en S1-13.

Tableau 17. Reformulations pertinentes sur le plan sémantique et interactionnel

\begin{tabular}{|l|l|l|l|}
\hline Séance & $\mathbf{N}^{\mathbf{0}}$ & Loc & Interventions \\
\hline 1 & 10 & Shona & $\begin{array}{l}\text { [...] il passe entre les jambes de monsieur Grégory et après Pierre il rentre } \\
\text { dans la remise }\end{array}$ \\
\hline 1 & 11 & Oudriss & après/ après Pierre/ Pierre et Romarin eh bien/ et après il a dit ouf/ \\
\hline 1 & 12 & M & quand est-ce qu'il a dit ouf \\
\hline 1 & 13 & Oudriss & eh bien il a couru et après et après il a dit ouf \\
\hline
\end{tabular}

La réussite de la reformulation adaptée ne se joue pas seulement dans la maitrise du «sens » des mots : Oudriss comprend fort bien la séquence restrictive citée plus haut puisqu'il la reformule de manière imagée et convaincante. Elle se joue de manière plus complexe dans l'articulation réussie d'une saisie (ce qui doit repris/dit du texte) d'une mise en mots (reprise ou reformulation) et d'un contexte qui assure au locuteur un gain sémantique et interactionnel. Ainsi, on voit Céline, qui est de niveau 3, introduire le mot terrier dans un contexte qui vient conclure doublement les interventions précédentes : en tant qu'elle répond à Ewan (" ensuite, il a arrêté de courir ») mais aussi en tant qu'elle clôt cette phase narrative par le retour au « terrier » à la fin de ces péripéties.

Tableau 18. Reformulations pertinentes sur le plan sémantique et interactionnel

\begin{tabular}{|l|l|l|l|}
\hline Séance & $\mathbf{N}^{\mathbf{0}}$ & Loc & Interventions \\
\hline 10 & 64 & Ewan & il a couru dans les jambes de monsieur Grégory \\
\hline 10 & 65 & M & Marjorie \\
\hline 10 & 66 & Marjorie & il a perdu ses deux chaussures \\
\hline 10 & 67 & M & Céline \\
\hline 10 & 68 & Céline & il a arrêté de courir dès qu'il est: dès qu'il est arrivé dans son terrier \\
\hline
\end{tabular}

La question ne porte donc pas sur la difficulté supposée des termes ${ }^{15}$ pas plus que sur les procédures qui sont assez proches entre les élèves de niveaux différents.

Reste un critère à prendre en compte qui est l'origine du terme repris. Remontons en quelque sorte en amont de ces procédures. Comment les élèves procèdent-ils pour opérer la «saisie lexicale » qui est l'origine de la reprise ou de la reformulation? 


\section{L'origine interactionnelle des reprises et des reformulations}

Nous avons distingué les reprises selon qu'elles apparaissaient pour la première fois dans la séance et ne pouvaient avoir que le texte original lu par l'enseignant comme source (Texte) de celles qui étaient précédées par de premières mentions dans les interventions d'autres élèves (Élèves) ou d'une intervention magistrale $(M)$, enfin nous avons comptabilisé les reprises d'une première mention quand elle était le fait d'un même élève (So) qui donc revenait au cours de la séance sur un thème qui en général lui tenait à cœur.

\section{Les reprises}

Quel que soit le niveau des élèves, dans la majorité des cas, le terme repris trouve son origine dans ce qu'ont déjà dit d'autres élèves (colonne grisée) et dans $36 \%$ à $39 \%$ des cas la reprise est faite à partir du texte (en gras). Dans $90 \%$ des cas donc, les élèves ont donc les mêmes stratégies dans le choix des sources de leurs reprises, adaptées ou essayées. Les dix pour cent restant se répartissent entre les reprises magistrales (colonne $\mathrm{M}^{16}$ ) ou des reprises de ce que l'élève a lui-même introduit dans une de ses précédentes interventions (colonne So).

Tableau 19. Pourcentages de l'origine des reprises selon le niveau des élèves

\begin{tabular}{|l|l|l|l|l|}
\hline & Élèves & Texte & So & M \\
\hline Niv. 1 & $53 \%$ & $\mathbf{3 9} \%$ & $6 \%$ & $2 \%$ \\
\hline Niv. 2 & $54 \%$ & $\mathbf{3 8} \%$ & $4 \%$ & $4 \%$ \\
\hline Niv. 3 & $54 \%$ & $\mathbf{3 6} \%$ & $4 \%$ & $6 \%$ \\
\hline
\end{tabular}

\section{Les reformulations}

Les élèves de niveau 3 (ci-dessous, tableau 21, en gras) sont moins autonomes que ceux de niveau 1 (cellules grisées) d'environ 10 points. Dans $44 \%$ des cas au lieu de $31 \%$ pour ceux de niveau 1 ils dépendent des autres élèves (colonne élèves), tandis que ceux de niveau 1 ont une plus grande capacité à trouver par eux-mêmes les termes dont ils ont besoin (colonne So).

Tableau 20. Pourcentages de l'origine des reformulations selon le niveau des élèves

\begin{tabular}{|l|l|l|l|l|}
\hline & Élèves & So & M & Total \\
\hline Niv.1 & $31 \%$ & $68 \%$ & $1 \%$ & $100 \%$ \\
\hline Niv. 2 & $40 \%$ & $60 \%$ & $0 \%$ & $100 \%$ \\
\hline Niv.3 & $\mathbf{4 4} \%$ & $\mathbf{5 6} \%$ & $0 \%$ & $100 \%$ \\
\hline
\end{tabular}

\section{Compétences des élèves de niveau 3}

41 Il n'y a donc pas de différence qualitative essentielle entre les élèves d'un niveau et d'un autre, sinon pour les élèves de niveau 3 une plus grande dépendance vis-à-vis des autres élèves auxquels ils empruntent plus souvent leurs reformulations. Comme nous l'avons 
déjà vu, (colonne grisée dans le tableau ci-dessous) on sait que les élèves de niveau 1 ont produit plus de la moitié des interventions de l'ensemble du corpus, tandis que les élèves des deux autres niveaux se partagent le reste, respectivement $29 \%$ et $21 \%$. Les différences entre les trois niveaux sont les mêmes si l'on ne considère que les interventions comportant un terme étudié (deuxième colonne). Par contre, on constate que le pourcentage des élèves de niveau 3 chute (17\%) lorsqu'on prend en compte les interventions comportant plusieurs termes étudiés que nous nommerons les interventions à haute densité lexicale.

Tableau . Pourcentages des interventions comportant un ou plusieurs TE selon les niveaux

\begin{tabular}{|l|l|l|l|}
\hline Interventions & Ensemble des interventions & Interventions avec un seul TE & Interventions avec plus d'un TE \\
\hline Niv. 1 & $50 \%$ & $51 \%$ & $\mathbf{5 2} \%$ \\
\hline Niv. 2 & $29 \%$ & $28 \%$ & $\mathbf{3 1} \%$ \\
\hline Niv. 3 & $21 \%$ & $21 \%$ & $\mathbf{1 7} \%$ \\
\hline
\end{tabular}

\section{La composition des interventions à plus d'un TE}

Lorsque l'élève fait une intervention à haute densité lexicale, il y a trois possibilités : soit il n'introduit que des termes nouveaux, soit il n'introduit que des termes déjà donnés, soit enfin il introduit des termes nouveaux et d'autres déjà donnés dans les interventions précédentes.

Le tableau des résultats bruts montre que ce sont ces interventions « mixtes » qui sont les plus nombreuses, en particulier pour les élèves de niveau 1 et 2 (cellules grisées). Pour les élèves de niveau 3, ce sont les interventions qui s'appuient sur ce qui a déjà été dit qui sont les plus nombreuses: on sait l'importance à ce niveau des reprises à partir des interventions déjà faites. Mais ils ne négligent pas d'apporter leur propre contribution aux échanges, ainsi que le montrent pour eux leurs résultats (en gras). Pourquoi ? Comment?

Tableau . Nombre d'interventions comportant plus d'un terme étudié selon les niveaux

\begin{tabular}{|l|c|c|c|c|}
\hline & Avec seulement des termes nouveaux & Avec seulement des termes déjà donnés & Interventions mixtes & Totaux \\
\hline Niv. 1 & 22 & 30 & 59 & 111 \\
\hline Niv. 2 & 18 & 22 & 25 & 65 \\
\hline Niv. 3 & $\mathbf{1 0}$ & $\mathbf{1 5}$ & $\mathbf{1 1}$ & $\mathbf{3 6}$ \\
\hline Totaux & 50 & 67 & 95 & 212 \\
\hline
\end{tabular}

La longueur moyenne de ces trois types d'interventions mesurée en nombre de mots nous donne une première indication.

Tableau . Longueur moyenne mesurée en nombre de mots des interventions comportant plusieurs TE

\begin{tabular}{|l|c|c|c|}
\hline & Avec seulement des termes nouveaux & Avec seulement des termes déjà donnés & Interventions mixtes \\
\hline Niv. 1 & 35 & 24 & 40 \\
\hline Niv. 2 & 21 & 25 & 29 \\
\hline Niv. 3 & $\mathbf{1 3}$ & $\mathbf{1 8}$ & $\mathbf{2 3}$ \\
\hline
\end{tabular}

Contrairement à ce qu'on aurait pu attendre, ce ne sont pas les interventions comportant seulement des termes déjà donnés qui sont les plus longues. Quand ils le peuvent, les 
élèves préfèrent apporter quelque chose de nouveau aux échanges. Ce sont donc les interventions mixtes qui sont les plus longues. C'est vrai pour les trois groupes.

Nous allons sur quelques exemples voir comment procèdent ces élèves qu'on catégorise généralement comme des petits parleurs.

\section{Reprises et reformulations dans les interventions mixtes}

Le corpus compte donc 11 interventions « mixtes " qui sont le fait de 8 élèves de niveau 3: Daoud (S1-42, S1-69), Keliane (S4-53, S4-55), Lili (S5-70), Daly (S6-15, S6-61), Ted (S8-94), Sonia (S9-26), Amid (S11-10) et Lise (S11-20). Nous allons étudier comment ils procèdent en étudiant quelques-unes des interventions de Daoud, Daly et Sonia.

\section{Daoud (S1-8). Reprises et reformulations}

Lors de la séance 1 , Daoud intervient trois fois $(8,42,69)$. Sa première intervention se situe donc au tout début de la séance. La voici.

Tableau . Daoud Intervention 8

\begin{tabular}{|l|l|l|l|}
\hline Séance & $\mathbf{N}^{\mathbf{o}}$ & Loc & Intervention \\
\hline 1 & 8 & Daoud & $\begin{array}{l}\text { eh bien la mère elle a dit vous ne pouvez jouer que dans le pré et elle a dit } \\
\text { n'allez surtout pas dans le potager }\end{array}$ \\
\hline
\end{tabular}

Elle porte sur les recommandations maternelles de la situation initiale. Les thèmes qu'elle aborde ont déjà été introduits par ses camarades (en gras ci-dessous en 2, 4 et 5).

Tableau . Daoud. Interventions précédentes

\begin{tabular}{|l|l|l|l|}
\hline Séance & $\mathbf{N}^{\mathbf{0}}$ & Loc & Interventions \\
\hline 1 & 1 & $\mathrm{M}$ & $\begin{array}{l}\text { on va raconter ensemble/ l'histoire dans l'ordre/ et on essaie de ne rien } \\
\text { oublier }\end{array}$ \\
\hline 1 & 2 & Mourad & $\begin{array}{l}\text { il y avait des lapins/ ils s'appelaient Romarin/ Capucine/ Neige/ Pierre/ } \\
\text { et après leur maman dit à ses lapereaux/ méfiez-vous/ je dois } \\
\text { m'absenter pour la journée }\end{array}$ \\
\hline 1 & 4 & Shona & $\begin{array}{l}\text { la maman elle a dit à ses petits lapins de ne pas y aller dans le } \\
\text { jotager parce que il y a monsieur Grégory/ elle a dit vous pouvez aller } \\
\text { que dans le potager de monsieur Grégory votre pauvre père qui est allé/ } \\
\text { il n'est jamais revenu et madame Grégory a fait un civet pour son repas }\end{array}$ \\
\hline 5 & Oudriss & $\begin{array}{l}\text { après Pierre/ après il a dit/ après il a fait atchoum/ et après il a fait et } \\
\text { après après Pierre et après Pierre il a été dans le potager et la maman } \\
\text { et la maman elle a dit ne passez pas dans le potager }\end{array}$ \\
\hline
\end{tabular}

Mais Daoud ne répète pas exactement ce qu'il vient d'entendre. 
Tableau . Daoud. Origine des termes étudiés

\begin{tabular}{|c|c|c|c|}
\hline Séance & $\mathrm{N}^{\circ}$ & Loc & Intervention \\
\hline 1 & 8 & Daoud & $\begin{array}{l}\text { eh bien la mère elle a dit vous ne pouvez jouer que dans le pré et elle } \\
\text { a dit n'allez surtout pas dans le potager }\end{array}$ \\
\hline
\end{tabular}

51 Il préfère désigner la lapine comme «la mère » plutôt que l'enfantin maman utilisé par Mourad et Shona et il assortit la recommandation d'un ne que exclusif. Ainsi, tout en s'appuyant entièrement sur les thèmes déjà introduits par ses camarades (cette intervention ne contient aucun TE nouveau à proprement parler), il réussit cependant à les présenter de manière personnelle. On peut constater que Mourad (S1-9) qui est un grand parleur et qui intervient immédiatement après, procède de la même manière en reformulant la recommandation maternelle (souligné).

Tableau . Daoud. Mourad enchaine sur l'intervention de Daoud

\begin{tabular}{|l|l|l|l|}
\hline Séance & $\mathbf{N}^{\mathbf{0}}$ & Loc & Interventions \\
\hline 1 & 4 & Shona & $\begin{array}{l}\text { la maman elle a dit à ses petits lapins de ne pas y aller dans le } \\
\text { potager parce que il y a monsieur Grégory/ elle a dit vous pouvez aller } \\
\text { jouer dans le pré/ mais surtout pas dans le potager parce que/ parce que } \\
\text { dans le potager de monsieur Grégory votre pauvre père qui est allé/ il } \\
\text { n'est jamais revenu et madame Grégory a fait un civet pour son } \\
\text { repas }\end{array}$ \\
\hline 1 & 8 & Daoud & $\begin{array}{l}\text { eh bien la mère elle a dit vous ne pouvez jouer que dans le pré et elle a } \\
\text { dit n'allez surtout pas dans le potager }\end{array}$ \\
\hline 1 & 9 & Mourad & $\begin{array}{l}\text { eh bien elle a dit surtout n'allez pas dans le potager/ votre pauvre } \\
\text { ùren n'est jamais revenu et elle a dit madame Grégory elle a fait } \\
\text { un civet pour son repas et la mère elle dit surtout restez très sage }\end{array}$ \\
\hline
\end{tabular}

Il commence en reprenant les derniers mots de Daoud, il enchaine sur deux thèmes donnés par Shona (4) à la fin de son intervention et conclut par l'introduction d'une recommandation encore inédite dans la séance: "et surtout restez très sages ", formulation dans laquelle on peut entendre un écho du «n'allez surtout pas dans le potager » que vient de prononcer Daoud.

\section{Daoud (S1-42). Empan conversationnel}

Daoud fait plus loin, une longue intervention en 42. Elle fait suite à cinq interventions auxquelles il va emprunter les premiers éléments de son intervention (en gras).

Tableau . Daoud. Intervention 42

\begin{tabular}{|l|l|l|l|}
\hline Séance & $\mathbf{N}^{\mathrm{o}}$ & Loc & Interventions \\
\hline
\end{tabular}




\begin{tabular}{|c|c|c|c|}
\hline 1 & 33 & Shona & $\begin{array}{l}\text { eh bien il a perdu sa chaussure dans les pommes de terre et après son } \\
\text { blouson il s'est accroché au fil et après à ce moment il enlève son gilet et } \\
\text { après il rentre dans la remise et sans réfléchir il saute dans l'arrosoir et il } \\
\text { a froid car il est à moitié dans l'eau et après il éternue et après monsieur } \\
\text { Grégory qui l'avait pas vu il se retourne et après il court/ il court/ il } \\
\text { arrive près d'un champ/ il rencontre un chat/ mais il n'ose pas lui } \\
\text { demander et après Pierre quand il est arrivé chez lui/ il monte dans sa } \\
\text { maison }\end{array}$ \\
\hline 1 & 35 & Sohaib & $\begin{array}{l}\text { il saute dans l'arrosoir et c'était vraiment pas une bonne idée parce que } \\
\text { c'était de l'eau glacée/ il a fait a/ atch/ atchoum/ et monsieur Grégory/ il } \\
\text { s'est retourné et après il a couru si vite }\end{array}$ \\
\hline 1 & 36 & Mourad & $\begin{array}{l}\text { monsieur Grégory il sait qu'il était dans l'arrosoir parce qu'il a éternué/ } \\
\text { parce que il était glacé/ Pierre/ et il s'est retourné monsieur Grégory }\end{array}$ \\
\hline 1 & 39 & Sihène & $\begin{array}{l}\text { eh bien quand Pierre est dans l'arrosoir/ eh bien il est/ il a encore couru } \\
\text { parce que monsieur Grégory il a retourné la terre et il l'a vu et Pierre il a } \\
\text { couru/ couru et monsieur Grégory il arrive et il a aussi couru couru pour } \\
\text { attraper Pierre et il veut le ramener dans sa maison/ monsieur Grégory/ } \\
\text { après monsieur Grégory il a couru pour le rattraper }\end{array}$ \\
\hline 1 & 40 & M & et après qu'est-ce qui se passe \\
\hline 1 & 42 & Daoud & $\begin{array}{l}\text { Pierre après/ quand il a éternué/ il a couru et après quand il a réussi à } \\
\text { rentrer à la maison/ sa maman elle/ elle lui a fait une tisane de camomille } \\
\text { et elle a dit tu passes une bonne nuit et après Pierre Romarin et Capucine/ } \\
\text { ils ont pris des mûres pour le diner }\end{array}$ \\
\hline 1 & 43 & Shona & et aussi ils ont bu le lait que leur maman a préparé \\
\hline
\end{tabular}

Mais sur cette base, Douad (42) va en quelque sorte développer toute la fin de l'histoire que Shona n'avait fait qu'esquisser; il introduit alors des TE nouveaux (en italiques) : il évoque la «tisane de camomille », le vœu de «bonne nuit » et enfin le diner de « mûres » cueillies par ses frères qu'il nomme avec une petite erreur « Pierre, Romain et Capucine ", il faut dire que Mourad (2) et Flora (3) les avaient déjà nommés mais au tout début de la séance, 40 tours de parole auparavant. Shona (43) ne se trompe pas sur l'intérêt, dans la séance, de cette intervention et de la nouveauté des informations apportées qu'elle complète en s'appuyant, elle, sur le texte initial.

\section{Sonia (S9-26). L'insertion dans les échanges}

Sonia qui a 4 ans et 4 mois, intervient dix fois pour de courtes interventions mais elle intervient régulièrement tout au long de la séance. L'ensemble ci-dessous montre bien son niveau de compétence syntaxique. Elle construit des propositions complètes qu'elle relie par un ET temporel ou logique selon les besoins, comme dans l'intervention 13 dont la valeur sémantique est assez ambigüe. 
Tableau . Sonia. Syntaxe additive

\begin{tabular}{|l|l|l|l|}
\hline Séance & $\mathbf{N}^{\mathbf{0}}$ & Loc & Interventions \\
\hline 9 & 5 & Sonia & Pierre il a couru dans le chemin qui l'a rentré \\
\hline 9 & 13 & Sonia & $\begin{array}{l}\text { la maman elle dit que je dois m'absenter vous pouvez aller dehors et Pierre } \\
\text { il n'a pas écouté et aussi Romarin Capucine et Neige ils ont écouté }\end{array}$ \\
\hline 9 & 18 & Sonia & la maman elle a pris du pain et du lait pour le repas/ \\
\hline 9 & 23 & Sonia & eh ben/ Pierre il a maintenant les chaussures et son gilet ils ont disparu \\
\hline
\end{tabular}

Malgré tout, Sonia travaille et ses interventions comme l'intervention 26 qui nous intéresse ici s'insèrent parfaitement dans les échanges entre élèves. Kader introduit des thèmes qui vont être repris et complétés par Sonia puis par Amélie et Luc.

Tableau . Sonia. Insertion dans l'échange

\begin{tabular}{|l|l|l|l|}
\hline Séance & $\mathbf{N}^{\mathbf{0}}$ & Loc & Interventions \\
\hline 9 & 24 & Kader & $\begin{array}{l}\text { il a saut Pierre il a sauté dans l'arrosoir mais l'eau elle était froide et } \\
\text { il a fait atchoum et après il a ressauté et recourt vers sa vitesse et il voit } \\
\text { un poisson mais il veut le manger mais quand même il ose pas le déranger }\end{array}$ \\
\hline 9 & 26 & Sonia & $\begin{array}{l}\text { monsieur Grégory Pierre il a sauté dans l'arrosoir et monsieur Grégory } \\
\text { s'est vite retourné vers l'arrosoir }\end{array}$ \\
\hline 9 & 27 & Amélie & $\begin{array}{l}\text { le le Pierre il a sauté dans l'arrosoir il a dit/ quelle erreur y avait y avait } \\
\text { beaucoup d'eau dans l'arrosoir et après il a Pierre il a fait atchoum et } \\
\text { monsieur Grégory il s'est retourné }\end{array}$ \\
\hline 28 & Luc & $\begin{array}{l}\text { // Pierre les il s'est attrapé par le bouton parce qu'il était dans le filet et } \\
\text { les oiseaux vite Pierre et Pierre il a sauté dans l'arrosoir et l'avait froid } \\
\text { et il a fait atchoum et puis monsieur Grégory il s'est retourné }\end{array}$ \\
\hline
\end{tabular}

Ainsi, Sonia s'appuie sur l'intervention de Kader qui vient d'introduire un élément nouveau: le saut dans l'arrosoir, elle le reprend en 26 (en gras) et poursuit en introduisant elle-même un élément nouveau qui est le déplacement de Monsieur Grégory (souligné) qui se retourne vers sa cachette. En 27, Amélie reprend ces deux éléments et introduit elle-même un élément nouveau, le commentaire venu du texte "quelle erreur " avec une explication intéressante de l'éternuement de Pierre : «il y avait beaucoup d'eau », imaginez un lapin dans un arrosoir! Enfin, Luc en 28, reprend ces différents éléments et propose une formulation sinon une explication nouvelle à cet éternuement « il avait froid».

Sonia est la seule de niveau 3, les autres sont de niveau 1 (Kader, Amélie) ou 2 (Luc). Ainsi donc, cet extrait de la séance 9 montre bien ce que les autres extraits illustraient déjà. Tous les élèves, quel que soit leur niveau, mobilisent les mêmes procédures 
fondamentales, la reprise et la reformulation qui portent sur des éléments déjà donnés qui leur sert de base pour l'introduction d'éléments nouveaux qui vont servir aux autres dans une spirale de construction discursive.

\section{Conclusion}

59 L'analyse de douze séances de langage en MS et GS a permis d'établir que dans un contexte favorable (choix pédagogiques et didactiques favorisant les prises de parole et l'écoute entre les membres du groupe), les élèves développent des procédures de reprises lexicales qui présentent des caractéristiques proches de ce que l'on a pu observer à l'écrit pour les difficultés rencontrées et les critères de sélection des termes mais qui se mettent en place selon des modalités propres à leur ancrage conversationnel.

60 L'analyse des différentes formes de reprises ou de reformulations montre que les difficultés rencontrées par les élèves tiennent d'abord à leur insertion syntagmatique des termes étudiés. Par contre, la grande majorité des formes retenues adaptées ou essayées (plus de $80 \%$ ) sont acceptables sur le plan sémantique et la rareté ou la nouveauté des termes n'est pas un obstacle. S'il y a un point commun à trouver à ces reprises ou ces reformulations réussies, c'est leur présence dans des interventions dont l'enjeu sémantique, sémiotique et interactionnel est clair pour les élèves.

61 L'éclairage nouveau que l'étude du corpus apporte est sans doute l'importance que joue le contexte polylogal dans lequel s'inscrivent ces interventions. Comme nous l'écrivions plus haut, la majorité des reprises ou des reformulations se font à partir de ce qu'ont déjà dit d'autres élèves, tandis que près de $40 \%$ des reprises ou des reformulations sont faites à partir du texte proprement dit.

Les trois groupes partagent donc la même compétence fondamentale, celle de réemployer des matériaux linguistiques déjà présents dans le contexte conversationnel. Tous sont capables, chacun à leur niveau, de s'appuyer dessus pour introduire des termes nouveaux dans des interventions à haute densité lexicale, même si cela suppose un allongement étonnant, parfois chaotique, de leurs énoncés. Ainsi l'apprentissage du lexique se trouvet-il ancré dans le développement des compétences langagières, inséparable de l'usage social du langage.

Dans quelles situations place-t-on l'élève à l'école maternelle? Combien de fois a-t-il l'occasion d'effectuer les opérations que nous venons d'évoquer pour acquérir un lexique nouveau?

La focalisation de l'étude sur un seul point, le lexique ou plus exactement sur ses conditions d'emploi, nous ramène à l'ensemble des paramètres des situations d'apprentissage proposées aux élèves. Les choix pédagogiques qui favorisent la prise de parole et l'écoute entre élèves comme le montre la dernière partie consacrée aux interventions à haute densité lexicale, les choix didactiques : un questionnement ouvert à propos d'un texte narratif adapté ${ }^{17}$, sont des éléments interdépendants de la réussite des élèves, de leurs interventions au sein du groupe et partant de l'apprentissage du lexique. 


\section{BIBLIOGRAPHIE}

ANCTIL, D. (2012). « Portraits des erreurs lexicales d'élèves de $3^{\mathrm{e}}$ secondaire en production écrite et proposition de pistes didactiques ». Pratiques 155-156, p. 7-30. En ligne : https:// journals.openedition.org/pratiques/3447.

CALAQUe, E. \& GROSSMANN, F. (2000). « Enseignement - apprentissage du lexique ». Lidil 21, p. 5-15. DUVIGNAU, K. \& GARCIA-DEBANC, Cl. (2008). « Un apprentissage du lexique verbal par proximité sémantique : quand la représentation lexicale facilite la tâche d'écriture ». In : Grossmann, F. \& Plane, S. (dirs), Les apprentissages lexicaux. Villeneuve d'Ascq : Presses universitaires du Septentrion, p. 17-42.

FLORIN, A. (1995). Parler ensemble en maternelle. La maîtrise de l'oral, l'initiation à l'écrit. Paris : Ellipses. FRANÇOIS, F. (1993). Pratiques de l'oral. Dialogue, jeux et variations des figures du sens. Paris : Nathan pédagogie.

FRANÇOIS, F., HUDELOT, C., \& SABEAU-JOUANNET, É. (1984). Conduites linguistiques chez le jeune enfant. Paris : Presses universitaires de France.

Grossmann, F. (2011). « Didactique du lexique : état des lieux et nouvelles orientations ». Pratiques 149-150, p. 163-183.

GROSSMANN, F. \& PLANE, S. (2008). Lexique et production verbale. Vers une meilleure intégration des apprentissages lexicaux. Villeneuve d'Ascq : Presses universitaires du Septentrion.

MARTINOT, C. (2010). « Reformulation et acquisition de la complexité linguistique ». Travaux de linguistique 61, p. 63-94. En ligne : https://www.cairn.info/revue-travaux-de-linguistique-2010-2page-63.htm.

MASSERON, C. (2005). « Indicateurs langagiers et stratégies scripturales. Du discours à la langue ». Pratiques 125-126, p. 205-249.

NONNON, É. (2012). « La didactique du français et l'enseignement du vocabulaire dans vingt ans de revues de didactique du français langue première ». Repères 46, p. 33-72. En ligne : https:// journals.openedition.org/reperes/88.

PÉROZ, P. (2014). « "parce que son papa, madame Grégory a fait un civet, un civet avec le papa". Sur les procédures d'apprentissage du lexique : reprise et reformulation en langage oral à l'école maternelle ", Congrès mondial de linguistique française, Berlin, 19-23 juil. En ligne : http://www.shsconferences.org/articles/shsconf/pdf/2014/05/shsconf_cmlf14_01221.pdf.

PÉROZ, P. (2015). « Apprentissage du langage oral à l'école maternelle. Quel modèle? ». In : Carton, F. et al. (dirs), Cultures de recherche en linguistique appliquée. Paris : Riveneuve Éd., p. 189-206.

PÉROZ, P. (2018). Pédagogie de l'écoute. Conduire et analyser une séance de langage. Paris: Hachette éducation.

STEINBACH-KOHLER, F. (2008). « Co-construction dans l'interaction en classe de FLE : De la dialogicité du langage vers la dialogicité de l'apprentissage ». TRANEL 48, p. 25-42. 


\section{NOTES}

1. Ministère de l'Éducation nationale, $B O$ spécial nº 2 du 26 mars 2015.

2. Le législateur écrivait: «Ces acquisitions décisives sont rendues possibles par l'attention que l'enseignant porte à chaque enfant, auquel il fournit les mots exacts en encourageant ses tentatives, et en reformulant ses essais pour lui faire entendre des modèles corrects. [...] c'est parce que les enfants entendent des phrases correctement construites et un vocabulaire précis qu'ils progressent dans leur propre maitrise de l'oral. » (Ministère de l'Éducation nationale, BO n 3, HS, 19 juin 2008).

3. Une première partie de cette étude qui portait alors sur 21 termes a été présentée en juillet 2014 lors du Congrès mondial de linguistique à Berlin (Péroz, 2014). À l'occasion de cette nouvelle communication, nous avons pu l'étendre à une cinquantaine de termes avec maintenant des résultats plus homogènes entre les différents groupes d'élèves.

4. "Réemploi » est utilisé ici pour désigner les reprises ou les reformulations de manière générique. Les deux termes sont définis un peu plus loin.

5. Pendant les séances de langage, ces termes n'ont pas fait l'objet de séances d'enseignement systématique mais ceux que les enseignants trouvaient difficiles comme pin (centenaire), pré ou civet ont été expliqués et commentés au début des premières séances avant la lecture du texte, en précisant aux élèves qu'ils allaient les retrouver dans l'histoire qu'on allait leur lire.

6. «L'approche cognitive du lexique et de son développement ne peut donc se faire en isolant le lexique des autres composantes du langage (syntaxe, sémantique, énonciation, discours), et il faut dépasser la centration sur le mot comme unité autonome » (Nonnon, 2012, p. 43).

7. Le critère retenu donne des résultats assez proches de celui proposé par A. Florin (1995) pour décompter « grands, moyens et petits parleurs » qui est la participation aux échanges sur la base du nombre de leurs interventions. Il en diffère cependant en cela qu'il donne une représentation plus précise de la part qui revient à chacun dans l'occupation de l'espace conversationnel.

8. Dans la séance trois, les élèves produisent des interventions nettement moins longues entre 5 et 7 mots selon le niveau, mais ils compensent cela par un nombre élevé d'interventions, 34 en moyenne par élève alors qu'il de 11,5 pour les élèves des autres séances.

9. L'expression de la mesure de la longueur moyenne est ramenée à l'unité. Une plus grande précision est possible mais relativement illusoire par rapport à la construction souvent hésitante des interventions orales.

10. 56 interventions faites par des élèves non identifiés notés Els et qui ont une longueur moyenne de 2,5 mots ne sont pas prises en compte dans ces calculs. Cela ne modifie pas les résultats présentés ici puisque trois interventions seulement de ce type comptent un TE en l'occurrence : mûres, pâté et méchant aux lignes 1-76, 2-51 et 3-397 du corpus.

11. On trouve le plus souvent une seule occurrence d'un des termes, exactement dans 543 interventions (1 seul mot étudié dans 1 intervention) et plus rarement plusieurs occurrences de termes différents dans une même intervention, exactement dans 212 interventions.

12. E. Nonnon (2012, p. 44) distingue entre « la paraphrase et la reformulation [qui] apparaissent comme centrales dans leurs stratégies lexicales». Nous ne faisons pas cette distinction qui renvoie à deux niveaux d'analyse linguistique différents dont la prise en compte n'est pas nécessaire dans le cadre de cette étude. Nous nous en tenons à un seul niveau, linguistique, où l'identification des unités est relativement simple. Nous distinguons deux procédures de réemploi, les «reprises » du TE à l'identique et les «reformulations" au sens où l'entend C. Martinot (2010), c'est-à-dire qu'au-delà du remplacement du TE par un autre terme, la nouvelle séquence conserve toujours une forme d'invariance sémantique par rapport au TE original. 
13. La construction est possible: "cuisiner un lapin en pâté, en civet», c'est-à-dire le transformer en civet ou en pâté. Mais le verbe original est « transformer » et non « cuisiner », car dans les faits ainsi que certains élèves le font remarquer c'est madame Grégory et non monsieur Grégory qui va « cuisiner» le lapin en question. C'est la raison pour laquelle nous catégorisons cette reprise comme « essayée » et non " adaptée ».

14. Les nombres donnés en pourcentages sont ramenés à l'unité pour faciliter la lecture et parce que descendre en dessous n'aurait pas nécessairement beaucoup de sens étant donné la taille relativement modeste du corpus sur lequel nous travaillons.

15. Du point de vue didactique, la difficulté du terme en soi est posée comme un préalable quand on aborde la question à partir de listes de fréquence.

16. L'enseignant est indirectement à l'origine des TE dans la mesure où il a oralisé le texte de l'histoire mais lors de la séance proprement dite, il est rare qu'il soit directement à la source d'une reprise (entre $2 \%$ et $6 \%$ des cas).

17. Avec des aménagements mineurs, ce texte a été utilisé comme support de séances de langage dans de nombreuses classes de grande section et en particulier dans des écoles en REP+ avec des résultats tout à fait satisfaisants, présentés dans P. Péroz (2018).

\section{RÉSUMÉS}

Comment les élèves d'école maternelle apprennent-ils le vocabulaire qu'on leur présente au fil des séances disciplinaires? La communication présente les résultats de l'analyse d'un corpus transcrit d'une quinzaine de séances de langage en grande et en moyenne section. Elle montre que les procédures de reprise ou de reformulation qui sont à l'origine de l'apprentissage du lexique reposent essentiellement sur des mécanismes interactionnels: reprise ajout et reformulation, quel que soit le niveau des élèves.

How do pre-primary school children learn the vocabulary they are taught during disciplineoriented work sessions? This paper presents the results of the analysis of a transcribed corpus of about fifteen language sessions in upper and medium classes. It shows that the error correction and reformulation procedures, which are the main reasons for lexicon learning, are essentially based on interactional mechanisms: correcting, adding and rephrasing, irrespective of the level of the pupils.

\section{INDEX}

Keywords : school language, learning of lexicon, discursive community, collaboration and oral language appropriation

Mots-clés : langage de l'école, apprentissage du lexique, communauté discursive, collaboration et apprentissage du langage oral 
AUTEUR

PIERRE PÉROZ

Université de Lorraine, CREM, EA 3476, F-57000, France 